\title{
Analysis of Causes of Death Among Brought-in-Dead Cases in a Third-Level Hospital in Lusaka, Republic of Zambia, Using the Tariff Method 2.0 for Verbal Autopsy: A Cross-Sectional Study
}

Yuta Yokobori ( $\sim$ horihori221@hotmail.com )

National Center for Global Health and Medicine, Japan https://orcid.org/0000-0002-1473-6583

Jun Matsuura

National Center for Global Health and Medicine, Japan

Yasuo Sugiura

National Center for Global Health and Medicine, Japan

Charles Mutemba

Adult Hospital, University Teaching Hospital, Zambia

Martin Nyahoda

Department of National Registration, Passport and Citizenship, Ministry of Home Affair, Zambia

Chomba Mwango

Department of National Registration, Passport and Citizenship, Ministry of Home Affair, Zambia

Lloyd Kazhumbula

Department of National Registration, Passport and Citizenship, Ministry of Home Affair, Zambia

Motoyuki Yuasa

Department of Public Health, Graduate School of Medicine, Juntendo University

Clarence Chiluba

Ministry of Health, Zambia

Research article

Keywords: Civil registration and vital statistics, Death, Africa, Zambia, International Classification of Disease, Automated Verbal Autopsy, Verbal Autopsy, , SmartVA

Posted Date: March 26th, 2020

DOI: https://doi.org/10.21203/rs.2.17243/v4

License: (c) (1) This work is licensed under a Creative Commons Attribution 4.0 International License. Read Full License

Version of Record: A version of this preprint was published at BMC Public Health on April 10th, 2020. See the published version at https://doi.org/10.1186/s12889-020-08575-y. 


\section{Abstract}

Background: Over one third of deaths in Zambian health facilities involve someone who has already died before arrival (i.e., Brough in Dead), and in most BiD cases, the CoD have not been fully analyzed. Therefore, this study was designed to evaluate the function of automated VA based on the Tariff Method 2.0 to identify the CoD among the BiD cases and the usefulness by comparing the data on the death notification form.

Methods: The target site was one third-level hospital in the Republic of Zambia's capital city. All BiD cases who reached the target health facility from January to August 2017 were included. The deceased's closest relatives were interviewed using a structured VA questionnaire and the data were analyzed using the SmartVA to determine the CoD at the individual and population level. The CoD were compared with description on the death notification forms by using t-test and Cohen's kappa coefficient.

Results: 1378 and 209 cases were included for persons aged 13 years and older (Adult) and those aged 1 month to 13 years old (Child), respectively. The top CoD for Adults were infectious diseases followed by noncommunicable diseases and that for Child were infectious diseases, followed by accidents. The proportion of cases with a determined CoD was significantly higher when using the SmartVA (75\% for Adult and $67 \%$ for Child) than the death notification form (61\%). A proportion (42.7\% for Adult and $46 \%$ for Child) of the CoDdetermined cases matched in both sources, with a low concordance rate for Adult (kappa coefficient $=0.1385$ ) and a good for Child(kappa coefficient $=0.635)$.

Conclusions: The CoD of the BiD cases were successfully analyzed using the SmartVA for the first time in Zambia. While there many erroneous descriptions on the death notification form, the SmartVA could determine the CoD among more BiD cases. Since the information on the death notification form is reflected in the national vital statistics, more accurate and complete CoD data are required. In order to strengthen the death registration system with accurate CoD, it will be useful to embed the SmartVA in Zambia's health information system.

\section{Background}

Information about deaths is essential for effective policy-making in order to address local demands in relation to various public health issues. The causes of death $(\mathrm{COD})$ information is especially significant for guiding health policy for the prioritization of health issues [1]. Ideally, all deaths should be registered and the CoD data should be obtained from accurate medical certification [2]. However, according to the World Health Statistics in 2015 [3], most low- and middle-income countries (LMIC) have a low death registration rate and inaccurate vital statistics [4]. In this context, strengthening death registration has attracted international attention as an important issue in the global public health field. The United Nations goal 16 of the sustainable development goals $[5,6]$ contains a target related to civil registration and vital statistics (CRVS) in order to promote human security.

The Republic of Zambia is one such country that is faced with substantial challenges to collecting accurate death information. According to the Central Statistics Office in Zambia, the death registration rate was $20 \%$ in 2016 [7]. One reason for the low registration rate is the large proportion of out-of-facility deaths. According to the Sample Vital Registration with Verbal Autopsy (SAVVY) which is standardized survey to investigate the CoDs of community death by using verbal autopsy, approximately $50 \%$ of the deceased died at home [8]. In addition, the health information system of the Zambian Ministry of Health in 2016 revealed that more than one third of death 
cases in health facilities died before their arrival, which are called brought-in-dead (BiD) cases. However, the CoD of these BiD cases have not yet been thoroughly analyzed. The background information of the BiD cases is one of the key factors for understanding the bottlenecks in the health system that obstruct the diseased from accessing the facilities before death. However, only a few publications have analyzed BiD cases in LMIC [9], including the African nations $[10,11]$.

In order to classify the CoD of these BiD cases, verbal autopsy (VA): a method of collecting information about symptoms and conditions for a deceased individual to determine his or her CoD, is now considered a realistic alternative to the medical certification of CoD [12]. The World Health Organization (WHO) has recommended using VA to strengthen the vital statistics regarding deaths to capture the $\mathrm{CoD}$ and trends in the national population [13]. While many countries [14-17] have incorporated VA into their official health information system, physician-based VA for all BiD cases may not be feasible in terms of the workload and costs. Therefore, automated VA is being developed to enable non-physician healthcare staff to make a reliable diagnosis of the $\mathrm{CoD}$ of BiD cases by using a computer program that can assign the most probable CoD by asking the deceased's closest relatives the relevant questions.

Among several types of automated VA program, the Tariff Method 2.0 is one of the standardized tools that can be used to predict individual CoD by estimating the cause specific mortality fraction (CSMF) that is based on a comparison with the Population Health Metrics Research Consortium (PHMRC) gold standard database [18]. Previous studies have shown that the Tariff Method 2.0 can satisfactorily diagnose the CoD from the data obtained from the structured VA questionnaires in terms of its validity [18-23]. The WHO recommends the Tariff Method 2.0 as one of the effective automated VA tools[19,20]. However, since none of the VA tools have been routinely used in Zambia, the CoD of most BiD cases have not been investigated in detail. Therefore, this study was designed to evaluate the function of automated VA based on the Tariff Method 2.0 to identify the CoD among the BiD cases who visited the main health facility in Zambia, and the usefulness by comparing the data with the written CoD information on the death notification form.

\section{Methods}

\section{Research Design}

This research was designed as Cross-Sectional Study to analyze causes of death among BiD cases in a third level hospital in Lusaka, Republic of Zambia.

\section{Target Site, Population, and Research Period}

The target site was a third-level hospital, the University Teaching Hospital (UTH), which is located in the capital of Zambia (Lusaka City). This city has a population of approximately 2.5 million. Since this health facility was the only third-level hospital in Lusaka that had a forensic doctor at the beginning of the research, most BiD cases in Lusaka were referred to the UTH. The target population was all BiD cases who reached the target health facility during the research period. BiD was defined as a death case who had already died before arrival at the hospital. The neonatal population aged 1 month and younger was excluded because neonatal death cases are usually transferred to a different ward in the UTH. The research period was for 4 months from May to August 
2017. While the data should be collected for 1 year because of seasonal changes in the epidemiological profile, the limitation of resources for the research restricted the research period.

\section{Data Collection}

Once the BiD cases reached the BiD ward at the UTH, the interviewers employed for this research interviewed the deceased's closest relatives using a paper-based questionnaire designed for the Tariff Method 2.0 for VA, which was developed by the WHO and Institute for Health Metrics and Evaluation (IHME); that is, the paper version of the PHMRC Shortened Questionnaire[24] which validity is discussed by Peter Serina et.al [18]. The researcher trained interviewers and made a 2-week pilot study before actual data collection. Then, the interviewers conducted the paper-based questionnaire by English and converted the data collected into an electronic format by using an application on an Android tablet called Open Data Kit (ODK) Collect[25]. The data were then stored in ODK Collect and transferred to the researchers' personal computers via the internet by using a cloud-based data management program called ODK Aggregate. In addition, the interviewers collected and recorded what was written about the $\mathrm{CoD}$ on the death notification form issued for the same $\mathrm{BiD}$ cases in order to compare the CoD with the results of the automated VA. The death notification form is an official template used to record the backgrounds of the death cases in Zambia, such as the name, address, age, and CoD certified by medical doctors. The relatives are required to submit the death notification form to the civil registration office in order to obtain the death registration, which is necessary to gain the burial permission. Usually, there is insufficient information to determine the CoD on the death notification form of the BiD cases.

\section{Data Analysis}

The researchers extracted the data from ODK Aggregate as Comma Separated Value (CSV) files and uploaded them into the SmartVA program [25], which is a computer program developed by the IHME to assign individual CoD by using the Tariff Method 2.0, in order to identify the top $10 \mathrm{CoD}$ of the adult and child BiD cases in the UTH. In order to automatically assign individual CoD, tariffs were created as CoD-specific normalized endorsement rates for each symptom reported in the PHMRC gold standard dataset. The tariff scores were calculated by summing all of the tariffs for the symptoms that were endorsed by the VA. Once the tariff scores were calculated for all of the samples in the dataset, they were compared to those whose true CoD was known from the PHMRC gold standard VA dataset. The CoD with the best tariff score, when compared to the gold standard VA, was then assigned using the Tariff Method 2.0's 34 categories for persons aged 13 years and older and 21 categories for those aged 1 month to 13 years [27]. In this research, persons for the cases aged 13 years old and older are called as "Adult" and those aged 1month to 13 years are called "Child". The CoD were categorized using the Tariff Method 2.0, which corresponds to the codes of the International Classification of Diseases-10 ${ }^{\text {th }}$ revision (ICD-10). If a VA in the input data has tariff scores that are significantly lower than all of the tariff scores in the PHMRC gold standard dataset, the CoD is marked as "undetermined" for the individual estimates. These causes are redistributed to the CSMF based on country-specific cause fractions for the population-level estimates.

The CoD obtained using the SmartVA were compared with those on the death notification form in terms of the CoD-determination rate and concordance rate. In order to compare the CoD on the death notification form, the descriptions about the CoD on the death notification form were assigned to an ICD-10 code by the research team, using as many categories as those assigned by the SmartVA. The CoD were marked as undetermined if 
the death notification form described only symptoms, such as fever, pain, and coughing; unspecific conditions, such as natural death, sudden death, and witchcraft; or other unidentifiable words.

All variables were processed using the STATA version 14 (StataCorp., College Station, TX, USA) and Microsoft Excel 2013 (Microsoft, Redmond, WA, USA) software. The statistical differences were estimated using the independent-samples $t$-test. The concordance rate was estimated using Cohen's kappa coefficient. For the VA, the SmartVA was utilized to estimate the CSMF by using the PHMRC Shortened Questionnaire and Tariff Method 2.0. The accepted level of significance is $p$-value $<0.05$ and the concordance level by kappa coefficient is considered excellent if 1.0-0.8, good if 0.6-0.8, moderate if 0.4-0.6 and poor if 0.6 and below [28].

\section{Results}

\section{The Characteristics of the BiD Cases}

Table 1 describes the characteristics of the adult BiD cases aged 13 years and older and child BiD cases aged 1 month to 13 years in the UTH. The total number of cases was 1378 for adults and 209 for children during the 4month research period from May to August 2017. There were 16 refusal cases in total. The most of reasons for refusals were respondents' busyness to handle the funerals and emotional strain on the deceased. These refusal cases were simply excluded from the analysis since the number was small. Regarding the sex, $62.8 \%$ of adult cases and $44.9 \%$ of child cases were male. The median ages of the adults and children were 42.0 and 2.08 years old, respectively. Figure 1 and Figure 2 present the age distribution according to the four age categories for the adult cases and by year for the child cases. Both figures show that there was not a normal distribution as the age of the adult cases had two peaks of distribution and the child BiD occurred more frequently in the younger ages. Most BiD cases originated from within Lusaka District.

\section{The CoD Among the Adult BiD Cases Aged 13 Years and Older}

Table 2 describes the top $10 \mathrm{CoD}$ among the adult BiD cases according to the SmartVA and death notification form. Among 1366 BiD cases in the UTH from May to August 2017, the top 10 CoD calculated by the SmartVA were, in order, acquired immunodeficiency syndrome (AIDS), stroke, tuberculosis (TB), suicide, diabetes mellitus (DM), other cardiovascular diseases (CVDs), pneumonia, epilepsy, acute myocardial infarction, and asthma. While two main infectious diseases each occupied one of the highest ranks of the $\mathrm{CoD}$, there were also several non-communicable diseases (NCDs). However, there were several discrepancies with the death notification form. Firstly, AIDS was not found in the top CoD; instead, TB and malaria ranked higher. Secondly, diarrhea/dysentery was replaced with pneumonia, and cancers and CVDs were ranked higher than stroke, epilepsy, and asthma.

If a case's tariff score was not significantly higher for a specific health condition than for other conditions, the SmartVA labeled it as an undetermined case. In addition, in the death notification form, some descriptions, such

as fever, pain, or natural death, could not be properly coded. Table 3 shows the number of determined CoD of the BiD cases according to the SmartVA and death notification form. The proportion of BiD cases whose CoD were determined was $61 \%$ for the death notification form and $75 \%$ for the SmartVA. The results of the statistical analysis showed that the SmartVA significantly improved the determination rate of the CoD compared with the death notification form. Table 4 presents the number of BiD cases whose CoD were not determined by the death notification form and SmartVA. The proportion of BiD cases with a determined CoD according to both methods 
was 686 (50.2\%). Regarding these cases, Table 4 shows that $43.3 \%$ of the CoD matched between the death notification form and SmartVA, and the concordance rate was low in term of the kappa coefficient $(0.08395 \%$ confidence interval [Cl]: 0.069-0.089).

Additional File 1 shows the distribution of the $\mathrm{CoD}$ among the adult $\mathrm{BiD}$ cases according to the four age categories: $13-19$ years, $20-44$ years, $45-59$ years, and 60 years and above. The proportion of undetermined cases was largest among people aged 13-19 years according to the death notification form (51.3\%) and smallest among those aged 45-59 years according to the SmartVA (19.9\%). Generally speaking, there was less malaria and more AIDS in the SmartVA group, compared with the death notification form, in the distribution of the $\mathrm{CoD}$ and the older the BiD cases became, the higher the number of NCDs there were in the top CoD. In addition, there were further differences between the CoD according to the SmartVA and death notification form. For example, the SmartVA identified more suicide cases among the younger population and more neurological conditions (stroke or epilepsy) and DM cases in all generations. Indeed, the concordance rates between the SmartVA and death notification form were weak among all of the age categories in terms of the kappa coefficient, whereas this coefficient was higher in the middle-aged categories (20-59 years) than in people aged $13-19$ years and 60 years and above.

\section{The CoD Among the Child BiD Cases Aged 1 Month to 13 Years}

Table 5 describes the top $10 \mathrm{CoD}$ among the adult BiD cases according to the SmartVA and death notification form. Among 205 BiD cases in the UTH from May to August 2017, the top 10 CoD calculated by the SmartVA were, in order, pneumonia, diarrhea/dysentery, human immunodeficiency virus (HIV)/AIDS, malaria, fires, drowning, other CVDs, meningitis, other defined CoD, and road traffic accident (RTA). Furthermore, infectious diseases, such as pneumonia, diarrhea/dysentery, and HIV/AIDS occupied the highest ranks of the CoD, followed by accidents, such as fire, drowning, and RTA. While the distribution of the CoD according to the death notification form was similar to that of the SmartVA, there were fewer HIV/AIDS cases on the death notification form and more epilepsy cases than meningitis cases. Table 6 shows how many of the CoD of the BiD cases were determined by the SmartVA and death notification form. The proportion of the BiD cases whose CoD were determined was $46 \%$ for the death notification form and $67 \%$ for the SmartVA. The statistical analysis indicated that the SmartVA significantly improved the determination rate of the CoD compared with the death notification form. Table 7 presents the number of $\mathrm{BiD}$ cases whose $\mathrm{CoD}$ were not determined by the death notification form and SmartVA. The proportion of BiD cases with determined CoD for both methods was 77 (37.6\%). Regarding these cases, Table 7 shows that $68.8 \%$ of the CoD were matched between the death notification form and SmartVA and the concordance rate was substantial in terms of the kappa coefficient $(0.635,95 \% \mathrm{Cl}$ : $0.548-$ 0.740).

\section{Discussion}

This research analyzed the CoD of 1366 adult and 205 child BiD cases who were brought into a third-level health facility in the capital city of Zambia by using an automated VA tool, called SmartVA, for the first time. The results indicate that the top CoD among the adult cases were infectious diseases, including AIDS, TB, and malaria, followed by NCDs, such as stroke, CVDs, and DM, and that those among the child cases were mainly infectious diseases, such as pneumonia, diarrhea, and malaria, followed by accidents. These trends are similar to the distribution of the CoD data of the Global Burden of Disease [26]. 
The comparison of the CoD between the SmartVA and death notification form showed that there are discrepancies in the distributions of the CoD among the adult cases; that is, there were fewer HIV/AIDS cases and more malaria cases in the death notification form. There were also fewer HIV/AIDS cases among the child cases in the death notification form. One possible reason is that the coders misclassified the CoD if a death case had two or more health conditions that lead to death. The WHO's instruction manual for ICD-10 coding [29] states in page 35, "When more than one condition is entered on the certificate, select the condition entered alone on the lowest used line of underlying causes of death only if it could have given rise to all the conditions entered above it." However, the coders for the death notification form may have reported the more immediate health conditions leading to death as the CoD. In that case, HIV/AIDS would be less likely to be selected as the final CoD.

In addition, there were more cases with an undetermined CoD on the death notification form compared with the SmartVA. This may be due to the healthcare staff's lack of knowledge about recording CoD. The symptoms, such as pain or fever, should not be noted as the CoD and unspecified health conditions, such as natural death or sudden death, should also be avoided. Thus, creating a standardized procedure for recording the CoD might be necessary.

Furthermore, the concordance rate between the SmartVA and death notification form was low especially among the adult BiD cases. This trend was the same among the different age categories, although the concordance rate was slightly higher in the middle-aged category (20-59 years) than in people aged 13-19 years and 60 years and above. One of the main reasons behind this discrepancy could be the larger number of undetermined cases and misclassification on the death notification form. Especially, the small number of AIDS cases as the CoD on the death notification form is not consistent with what is already known about this disease burden in Zambia [27]. Since these $C o D$ on the death notification form are reflected in the national vital statistics data, it is a problem that the actual data reported to the Civil Registration Office are skewed. The establishment of a system to improve the accuracy of the CoD among the BiD cases is necessary to capture the accurate vital statistics through the CRVS in a timely manner. Automated VA using the SmartVA is one option for strengthening the vital statistics since it can improve the quality of the determination of the individual $\mathrm{COD}$ and can be used to estimate the population level of the CoD from all samples.

There are several limitations in this research. Firstly, the research period in the UTH was only 4 months because of financial and human resource constraints. However, seasonal fluctuation needs to be considered for some health conditions, such as malaria, diarrhea, and probably some NCDs. Therefore, the results of this research cannot be completely generalized as annual data. However, since most health conditions do not vary by month, according to the existing health information system, these conditions that do not exhibit seasonal differences can be generalized as annual data. In addition, regarding generalizability, another limitation is that this research included the death cases brought to hospital, but not the death cases at home. In order to interpret burden of diseases in Lusaka, further research should be required in the future.

Secondly, there were several BiD cases in the UTH that were not included in this research. For example, some of children BiD cases were not covered because the UTH has another specific ward for children cases and some of these BiD cases were directly carried to the children wards. Such BiD cases in the children wards were unfortunately not well recorded. Furthermore, neonatal BiD cases were not captured because most of them were dealt with as hospital deaths once they were delivered to the neonatal intensive care unit. However, since most 
of the child BiD cases that were older than 1 month were directly carried to the BiD ward, this research did not just cover neonatal BiD cases. It is desirable to conduct another research study to investigate neonatal BiD cases in the UTH.

Thirdly, there was a substantial number of BiD cases with an undetermined CoD, even when using the SmartVA, although its determination rate was significantly better than that of the death notification form. When the SmartVA was used to analyze the top $10 \mathrm{CoD}$ among the same research samples by calculating the CSMF as the population level, there were discrepancies in the distribution compared to the results of the individual analysis in terms of there being more malaria and more other NCDs (Additional File 2). These differences may derive from the large number of undetermined cases, which account for approximately one fourth of the samples. In order to make the results reliable, the computer algorithm that determines the CoD should be improved to reduce the number of undetermined BiD cases in the future.

Lastly, the validity of the Tariff Method 2.0 for identifying the CoD by using the SmartVA should be considered more. According to Serina et al. [10], the chance-corrected concordance used to assess the extent to which the Tariff Method 2.0 correctly predicts an individual's CoD and the CMSF's accuracy for measuring the performance at the population level, when applied to the shortened version of the PHMRC Questionnaire, were $50.0 \%$ and $76.6 \%$ respectively for the adult population. However, since this validity was investigated by comparing it with the gold standard dataset [19], the applicability to the Zambian setting should be scrutinized by investigating the actual data. In addition, new automated VA tools have been developed, such as the InterVA5 [30], Naïve Bayes Classifier [31, 32], SilicoVA [33], and so on. We also need to investigate the functionality, feasibility, and validity of these new tools. In the future, a validation study that compares the CoD by using an automated VA tool with those determined by an actual full autopsy is required to estimate the true burden of diseases in Zambia.

\section{Conclusions}

By using an automated VA tool (i.e., the SmartVA that is based on the Tariff Method 2.0), the CoD of the BiD cases in the UTH have been successfully analyzed for the first time in Zambia. The SmartVA can determine the CoD of more BiD cases, when compared to the death notification form. In addition, the distribution of the CoD was inconsistent because there was a large number of erroneous descriptions of the CoD on the death notification form. Since these CoD on the death notification form are reflected in the national vital statistics data, it is a problem that the actual data reported to the Civil Registration Office are skewed. In order to strengthen the death registration system with accurate CoD, it will be useful to embed the automated VA into Zambia's health information system. However, further investigation into the validity of the CoD identified by the automated VA should be considered in the future.

\section{Declarations}

\section{Ethics approval and consent to participate}

Ethical approval was obtained from the University of Zambia's Biomedical Research Ethics Committee (ref: 01812-16) and the Ethics Committee of the National Center for Global Health and Medicine in Japan (ref: NCGM-G002167-00). Written informed consent was obtained from the closest relatives of the deceased. 


\section{Consent for publication}

Not applicable

\section{Availability of data and materials}

The datasets generated and/or analyzed during the current study are not publicly available due to an ethical restriction (patient confidentiality) but are available from the corresponding author on reasonable request

\section{Competing interests}

The authors declare that they have no competing interests

\section{Funding}

This work was also funded by Research Fund of Ministry of Health, Labour and Welfare (MHLW) in Japan for data collection, analysis and publication. The MHLW Research Fund provided supports for design of the study and collection, analysis and interpretation of data as well as necessary procedure for publication.

\section{Authors' information}

\section{Affiliations}

National Center for Global Health and Medicine, Tokyo, Japan. Yuta Yokobori, Jun Matsuura, Yasuo Sugiura Ministry of Health, Lusaka, Zambia. Clarence Chiluba

Adult Hospital, University Teaching Hospital, Lusaka, Zambia. Charles Mutemba

Department of National Registration, Passport \& Citizenship, Ministry of Home Affairs, Lusaka, Zambia. Martin Nyahoda, Chomba Mwango, Lloyd Kazhumbula

Department of Public Health, Graduate School of Medicine, Juntendo University, Tokyo, Japan. Motoyuki Yuasa

\section{Authors' contributions}

YY and MJ conducted writing proposal, data collection, data analysis, writing manuscript. CC contributed to first idea, writing methodology. SJ reviewed data analysis, final edition, native translating. CM and LK directed data collection for study. MN and YM reviewed the drafted manuscripts and revised them if necessary. All authors have read and approved the manuscript, and ensure that this is the case.

\section{Corresponding author}

Correspondence to Yuta Yokobori

\section{Acknowledgments}

The authors thank the Ministry of Health in Zambia, Dr. Kennedy Malama, Dr. Consity Mwale, Dr. John Musuku, Dr. Alex Makupe, Ms. Alice Zimba, Ms. Gift Izukanji, Mr. Ernest Matafwali, Mr. Justine Katukula for their help in 
accessing medical records needed for this study, data collection and data recording.

\section{Abbreviations}

AIDS: acquired immunodeficiency syndrome, AMl: acute myocardial infarction, BiD: brought in dead, Cl: confidence interval, CoD: causes of death, CRVS: civil registration and vital statistics, CSMF: cause specific mortality fraction, CVDs: cardiovascular diseases, DM: diabetes mellitus, HIV: human immunodeficiency virus, ICD-10: International Classification of Diseases-10 ${ }^{\text {th }}$ revision, IHD: Ischemic Heart Disease, IHME: Institute for Health Metrics and Evaluation, LMIC: low- and middle-income countries, NCDs: non-communicable diseases, ODK: Open Data Kit, PHMRC: Population Health Metrics Research Consortium, RTA: road traffic accident, TB: tuberculosis, UTH: University Teaching Hospital, VA: verbal autopsy, WHO: World Health Organization.

\section{References}

1. Mathers CD et al. Counting the dead and what they died from: an assessment of the global status of cause of death data. Bull World Health Organ. 2005; 83:171-7

2. Mahapatra P et al. Civil registration systems and vital statistics: successes and missed opportunities. Lancet. 2007; 370: 1653-63.

3. World Health Organization. World Health Statistics 2015. Geneva: WHO; 2015.

4. Phillips $D$ et al. A composite metric for assessing data on mortality and cause of death: the vital statistics performance index. Popul Health Metr.2014;12:14.

5. United Nations. The road to dignity by 2030: ending poverty, transforming all lives and protecting the planet synthesis report of the secretary-general on the post-2015 agenda. New York: UN; 2014.

6. Open Working Group for the UN General Assembly. Open working group proposal for sustainable development goals. New York: OWG; 2014.

7. Central Statistical Office of Zambia. 2016 Vital statistics report. Lusaka: Ministry of Home Affair of Zambia; 2019.

8. Central Statistical Office of Zambia. 2015/16 Zambia sample vital registration with verbal autopsy report. Lusaka: Ministry of Home Affair of Zambia; 2018.

9. Munawar Hursheed et al. Dead on arrival in a low-income country: results from a multicenter study in Pakistan. BMC Emergency Medicine. 2015; 15(2): S8

10. Khaled Bensaid et al. Verbal/social autopsy in Niger 2012-2013: A new tool for a better understanding of the neonatal and child mortality situation. Journal of Global Health. 2016; 6:1.

11. Arthur Mpimbaza et al. Verbal autopsy: evaluation of methods to certify causes of death in Uganda. PLOS ONE. 2015; 10(6): e0128801.

12. D'Ambruoso $L$ et al. Thecase for verbal autopsy in health systems strengthening. Lancet Glob Health. 2016; $11: 17$

13. World Health Organization, Verbal autopsy standards: ascertaining and attributing case of death. Geneva: WHO; 2007.

14. Indian Council of Medical Research. Study on causes of death by verbal autopsy in India. New Delhi: ICMR; 2009. 
15. Franca $E$ et al. Use of verbal autopsy in a national health information system: effects of the investigation of ill-defined causes of death on proportional mortality due to injury in small municipalities in Brazil. Popul Health Metr. 2011; 9:39.

16. Baqui AH et al. Causes of childhood death in Bangladesh: results of a nationwide verbal autopsy study. Bull World Health Organ. 1998; 76(2):161-71

17. Dharmaratne SD et al. Opportunities and challenges fir verbal autopsy in the national Death Registration System in Sri Lanka: past and future. Popul Health Metr. 2011; 9:21.

18. Peter Serina et al. A shortened verbal autopsy instrument for use in routine mortality surveillance systems. BMC Medicine. 2015; 13:302.

19. Spencer $L$ James et al. Performance of the Tariff Method: validation of a simple additive algorithm for analysis of verbal autopsies. Population Health Metrics. 2011; 9:31.

20. World Health Organization. Verbal autopsy standards: ascertaining and attributing cause of death. Available from https://www.who.int/healthinfo/statistics/verbalautopsystandards/en/.

21. Christopher JL Murray et al. Population Health Metrics Research Consortium gold standard verbal autopsy validation study: design, implementation, and development of analysis datasets. Population Health Metrics. $2011 ; 9: 27$.

22. Peter Serina et al. Improving performance of the Tariff Method for assigning causes of death to verbal autopsies. BMC Medicine. 2015; 13:

23. Weldearegawi e al. How much do the physician review and InterVA model agree in determining causes of death? A comparative analysis of deaths in rural Ethiopia. BMC Public Health. 2015;15(1): 669.

24. Institute of Health Metrics and Evaluation. Paper version of PHMRC Shortened Questionnaire. Available from

http://www.healthdata.org/sites/default/files/files/Tools/SmartVA/2015/PHMRC\%20Shortened\%20VAI_allmodules_2015.zip.

25. Open Data Kit Documentation. ODK collect. Available from https://docs.opendatakit.org/collect-intro/.

26. Institute of Health Metrics and Evaluation. Global Burden of Disease in Zambia. Available from healthdata.org/zambia.

27. Institute of Health Metrics and Evaluation. SmartVA Application. Available from http://www.healthdata.org/sites/default/files/files/Tools/SmartVA/2018/SmartVA-Analyze_2.0.0.exe.

28. Mary L. Interrater reliability: the kappa statistic. Biochem Med. 2012; 22(3): 276-282

29. World Health Organization. International statistical classification of diseases and related health problems.10th revision, volume 2 instruction manual edition 2010. Geneva: WHO; 2010.

30. Byass $P$ et al. An integrated approach to processing WHO-2016 verbal autopsy data; the InterVA-5 model. BMC Medicine. 2019; 17(1):102.

31. Miasnikof et al. Naïve Bayes classifiers for verbal autopsies: comparison to physician-based classification for 21000 child and adult deaths. BMC Medicine. 2015; 13:286.

32. Murtaza et al. Automated verbal autopsy classification: using one-against-all ensemble method and Naïve Bayes classifier. Gates Open Res. 2019; 2: 63.

33. Flaxman AD et al. Performance of InSilicoVA for assigning causes of death to verbal autopsies: multisite validation study using clinical diagnostic gold standards. BMC Medicine. 2018; 16(1):56. 


\section{Additional File Legends}

Additional File 1: Additional File 1.docx: Top 10 causes of death among adult brought-in-dead cases by agecategories during research period by SmartVA and Death Notification Form

Additional File 2: Additional File 2.docx: Top 10 causes of death according to the individual level and population level analysis

\section{Tables}

Table 1: Characteristics of the brought-in-dead adult and child cases in the University Teaching Hospital.

\begin{tabular}{lll}
\hline & Adult & Child \\
\hline Total Number & 1378 & 209 \\
Number of Refusals & 12 & 4 \\
\hline Male (\%) (95\% CI) & $62.8(60.2-65.4)$ & $44.9(38.0-51.7)$ \\
\hline Age (median year: 25-75th percentile) & $42.0(34.0-65.0)$ & $0.88(0.42-3.0)$ \\
\hline Origins of the Participants & & \\
\hline Within Lusaka & 1299 & 190 \\
Outside of Lusaka & 44 & 5 \\
\hline Unknown Origin & 23 & 10 \\
\hline
\end{tabular}


Table 2: Top 10 Causes of deaths among the brought-in-dead adult cases in the University Teaching Hospital during research period by SmartVA and Death Notification Form

\begin{tabular}{|cccc|cccc}
\hline \multicolumn{4}{c}{ SmartVA (N=1366) } & \multicolumn{3}{c}{ Death Notification Form (N=1366) } \\
\hline Rank & Cause of Death & No & $\%$ & Rank & Cause of Death & No & Un \\
\hline & Undetermined & 344 & $25.2 \%$ & & Undetermined & 526 & $38.5 \%$ \\
\hline 1 & HIV/AIDS & 278 & $20.4 \%$ & 1 & TB & 239 & $17.5 \%$ \\
3 & Stroke & 134 & $9.8 \%$ & 2 & Other CVDs & 148 & $10.8 \%$ \\
4 & TB & 87 & $6.4 \%$ & 3 & Malaria & 75 & $5.5 \%$ \\
5 & Suicide & 68 & $5.0 \%$ & 4 & Stroke & 59 & $4.3 \%$ \\
6 & DM & 67 & $4.9 \%$ & 5 & Other Cancers & 45 & $3.3 \%$ \\
7 & Other CVDs & 57 & $4.2 \%$ & 6 & Suicide & 33 & $2.4 \%$ \\
8 & Pneumonia & 52 & $3.8 \%$ & 7 & Diarrhea/Dysentery & 31 & $2.3 \%$ \\
9 & Epilepsy & 38 & $2.8 \%$ & 8 & Other NCDs & 28 & $2.0 \%$ \\
10 & IHD/AMI & 26 & $1.9 \%$ & 9 & DM & 25 & $1.8 \%$ \\
\hline
\end{tabular}

NB: TB: tuberculosis, DM: diabetes mellitus, CVDs: cardiovascular diseases, AMI: acute myocardial infarction, IHD: Ischemic Heart Disease, NCDs: non-communicable diseases, RTA: road traffic accident.

Table 3: Number of the brought-in-dead adult cases with determined causes of death by both of Death Notification Form and SmartVA in the University Teaching Hospital.

\begin{tabular}{lccccc}
\hline & No. of Cases With Determined CoDs & Mean & 95\% CI & $P$-value \\
\hline Death Notification Form & 840 & 0.61 & $0.589-0.641$ & \\
SmartVA & 1021 & 0.75 & $0.724-0.771$ & $<0.05$ \\
\hline
\end{tabular}

Table 4: The number of undetermined cases among the brought-in-dead adult cases and the concordance rate of CoDs among the cases with determined CoDs by both of Death Notification Form and SmartVA in the University Teaching Hospital 


\begin{tabular}{|c|c|c|c|}
\hline \multicolumn{3}{|c|}{ Number } & Percentage \\
\hline \multicolumn{2}{|l|}{ Both Undetermined } & 191 & $14.0 \%$ \\
\hline \multicolumn{2}{|c|}{ Undetermined only by Death Notification Form } & 335 & $24.5 \%$ \\
\hline \multicolumn{2}{|c|}{ Undetermined only by SmartVA } & 154 & $11.3 \%$ \\
\hline \multirow[t]{4}{*}{ Both Determined } & & 686 & $50.2 \%$ \\
\hline & Matched CoDs between both methods & 297 & $43.3 \%$ \\
\hline & Unmatched CoDs between both methods & 389 & $56.7 \%$ \\
\hline & Kappa coefficient & \multicolumn{2}{|c|}{0.083 (95\% CI: 0.069-0.086) } \\
\hline
\end{tabular}

NB: CoDs: Cause of Deaths

Table 5: Top 10 Causes of deaths among the brought-in-dead child cases in the University Teaching Hospital during research period by SmartVA and Death Notification Form

\begin{tabular}{|c|c|c|c|c|c|c|c|}
\hline \multicolumn{4}{|c|}{ SmartVA $(\mathrm{N}=205)$} & \multicolumn{4}{|c|}{ Death Notification Form $(\mathrm{N}=205)$} \\
\hline Rank & Cause of Death & No. & $\%$ & Rank & Cause of Death & No. & $\%$ \\
\hline & Undetermined & 68 & $33.2 \%$ & & Undetermined & 111 & $54.1 \%$ \\
\hline 1 & Pneumonia & 36 & $17.6 \%$ & 1 & Diarrhea/Dysentery & 18 & $8.8 \%$ \\
\hline 2 & Diarrhea/Dysentery & 32 & $15.6 \%$ & 2 & Other Defined CoD & 14 & $6.8 \%$ \\
\hline 3 & HIV/AIDS & 25 & $12.2 \%$ & 3 & Pneumonia & 10 & $4.9 \%$ \\
\hline 4 & Malaria & 5 & $2.4 \%$ & 4 & Malaria & 9 & $4.4 \%$ \\
\hline 5 & Fires & 5 & $2.4 \%$ & 5 & RTA & 7 & $3.4 \%$ \\
\hline 6 & Drowning & 5 & $2.4 \%$ & 6 & Drowning & 7 & $3.4 \%$ \\
\hline 7 & Other CVDs & 5 & $2.4 \%$ & 7 & Fires & 6 & $2.9 \%$ \\
\hline 8 & Meningitis & 4 & $2.0 \%$ & 8 & Other CVDs & 6 & $2.9 \%$ \\
\hline 9 & Other Defined CoD & 4 & $2.0 \%$ & 9 & Epilepsy & 5 & $2.4 \%$ \\
\hline 10 & RTA & 4 & $2.0 \%$ & 10 & Other Cancers & 5 & $2.4 \%$ \\
\hline
\end{tabular}

NB: CVDs: cardiovascular diseases, RTA: road traffic accident. 
Table 6: Number of the brought-in-dead child cases with determined causes of death by both of Death Notification Form and SmartVA in the University Teaching Hospital.

\begin{tabular}{lcccc}
\hline & No. of Cases With Determined CoDs & Mean & $95 \%$ CI & $P$-value \\
\hline Death Notification Form & 94 & 0.46 & $0.390-0.527$ & \\
SmartVA & 137 & 0.67 & $0.603-0.733$ & $<0.05$ \\
\hline
\end{tabular}

Table 7: The number of undetermined cases among the brought-in-dead child cases and the concordance rate of CoDs among the cases with determined CoDs by both of Death Notification Form and SmartVA in the University Teaching Hospital

\begin{tabular}{|c|c|c|c|}
\hline \multicolumn{3}{|c|}{ Number } & Percentage \\
\hline \multicolumn{2}{|c|}{ Both Undetermined } & 51 & $24.9 \%$ \\
\hline \multicolumn{2}{|c|}{ Undetermined only by Death Notification Form } & 17 & $33.2 \%$ \\
\hline \multicolumn{2}{|c|}{ Undetermined only by SmartVA } & 60 & $62.4 \%$ \\
\hline \multicolumn{2}{|l|}{ Both Determined } & 77 & $37.6 \%$ \\
\hline & Matched CoDs between both methods & 53 & $68.8 \%$ \\
\hline & Unmatched CoDs between both methods & 24 & $31.2 \%$ \\
\hline & Kappa coefficient & \multicolumn{2}{|c|}{0.635 (95\% CI: 0.548-0.740) } \\
\hline
\end{tabular}

NB: CoDs: Cause of Deaths

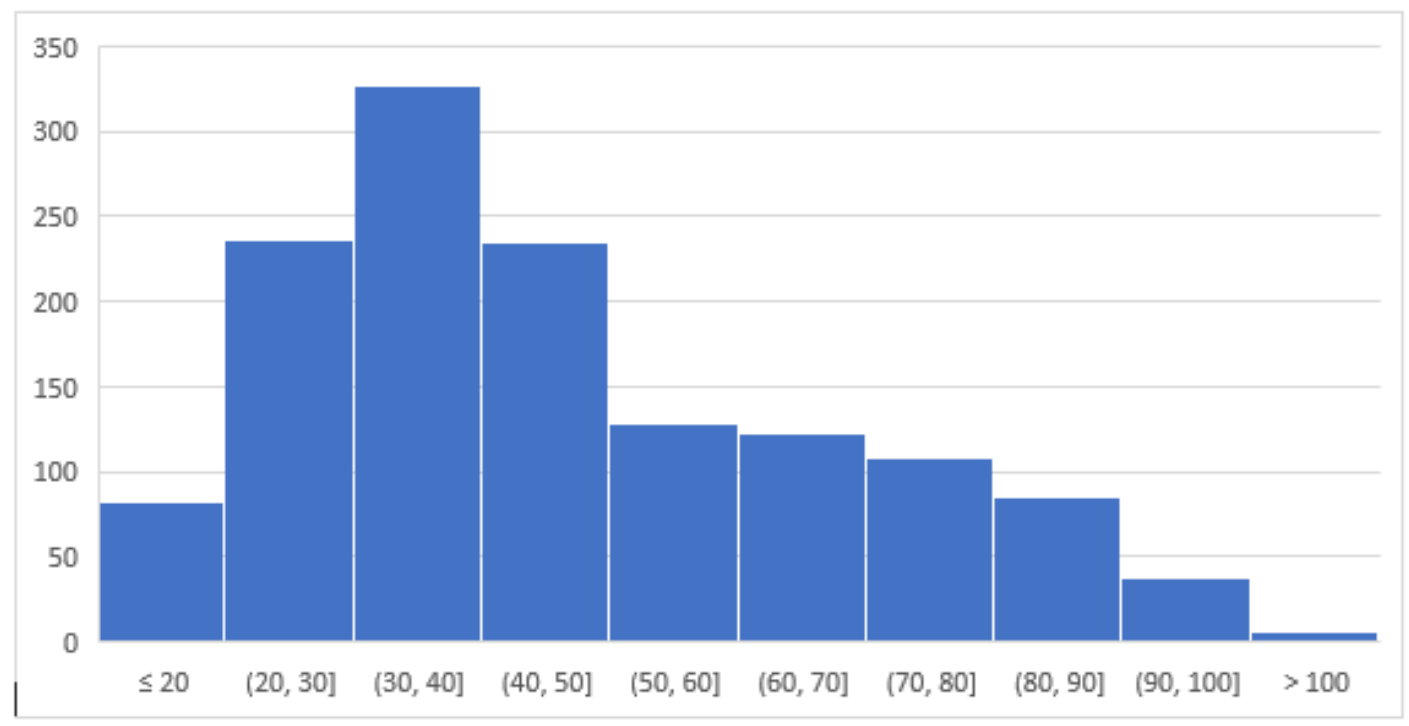

\section{Figure 1}

Age distribution of the adult brought-in-dead cases who were aged 13 years and older. The data were collected in the University Teaching Hospital during the research period. 


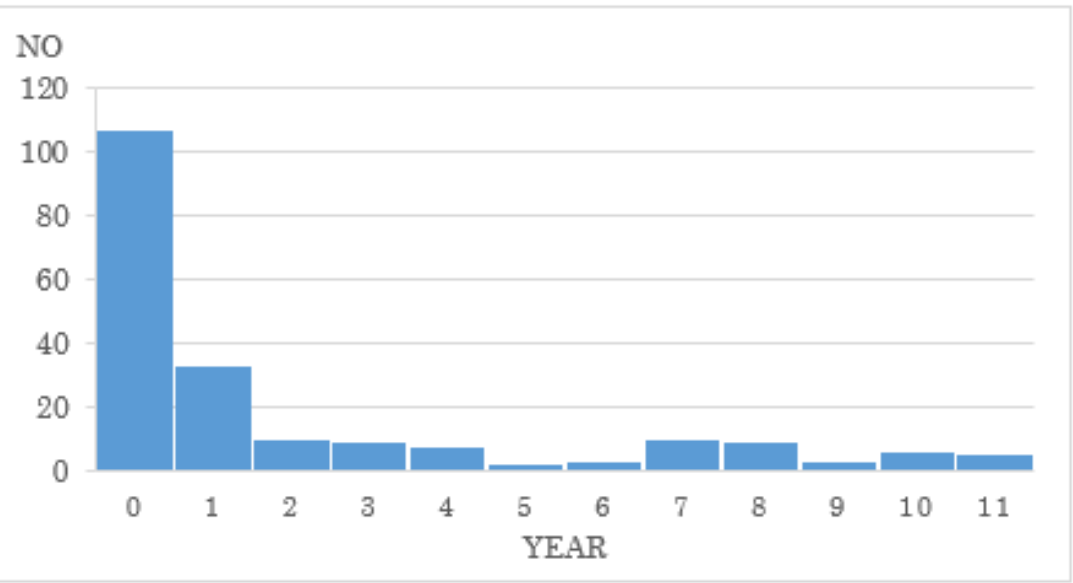

Figure 2

Age distribution of the child brought-in-dead cases who were aged 1 month to 13 years. The data were collected in the University Teaching Hospital during the research period.

\section{Supplementary Files}

This is a list of supplementary files associated with this preprint. Click to download.

- STROBEchecklistcrosssectional.doc

- AdditionalFile1rev.docx

- AdditionalFile2rev.docx 